\title{
The Central Dynamics of Blue Low Surface Brightness Galaxies
}

\author{
Erik Zackrisson and Nils Bergvall \\ Uppsala Astronomical Observatory, Box 515, SE 75120 Uppsala, \\ Sweden
}

\begin{abstract}
We use optical, long-slit rotation curves to derive the slope of the central density profile in three blue disk galaxies with very faint central surface brightness values. We find the result to be in conflict with current cold dark matter predictions and to lend further support for pseudo-isothermal spheres as superior models for the dark halos of galaxies.
\end{abstract}

\section{Introduction}

The halo mass density profiles predicted by cold dark matter (CDM) scenarios for structure formation (e.g. Navarro, Frenk, \& White 1996) appear to be in conflict with the profiles observed in the centres of low surface brightness galaxies (e.g. de Blok, Bosma, \& McGaugh 2003). This may pose a serious problem for the CDM paradigm, provided that dark matter really does dominate the dynamics in the central regions of these objects (as questioned by e.g. Fuchs 2003) and that the observations available are not subject to serious systematic errors (e.g. Swaters et al. 2003).

\section{Observations and Results}

Long-slit observations with the FORS2 spectrograph at the VLT have been used to derive optical rotation curves of six blue $(B-R<0.6)$ disk galaxies with very low central surface brightness values. Since blue colours are believed to minimize the $M / L$-ratio of the stellar population (Bell $\&$ de Jong 2001) and low central surface brightness values to maximize the dynamical M/L-ratio (McGaugh \& de Blok 1998), one may at least naively expect these selection criteria to minimize the influence of baryons on the central dynamics of these objects. Images obtained in $B, V$ and $I$ will be analyzed with the aid of spectral evolutionary models to constrain the allowed range of $M / L$-values allowed in the stellar populations of these particular objects.

In Figure 1 we present the outcome of the preliminary analysis of the slope of the central density profile in three of our target galaxies: ESO 031-13, ESO 46232 and ESO 548-09. The central surface brightness values of these objects (after correction for inclination and Galactic extinction) are $\mu_{0, B}=24.2,24.0$ and 24.5 respectively. Confirming most previous measurements, our data disfavours the CDM prediction and is consistent with the average $\alpha=-0.2 \pm 0.2$ derived by 


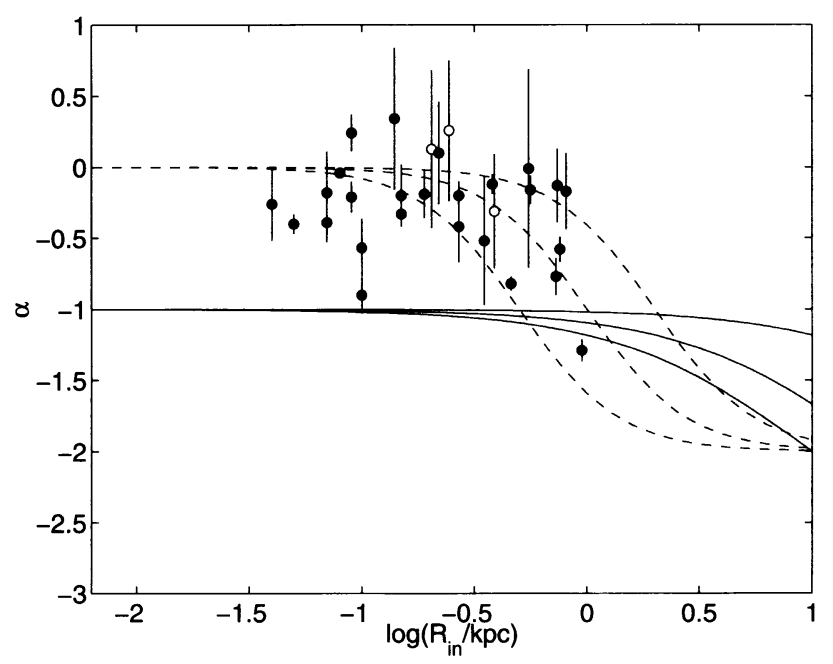

Figure 1. The power-law slopes, $\alpha$, of the central mass-density profiles, $\rho \propto r^{\alpha}$ derived from the five innermost data points of the rotation curves of our three target objects (open symbols). Filled symbols indicate the de Blok \& Bosma (2002) measurements. $R_{\text {in }}$ represents the radius of the innermost data point used in the fit. The three dashed lines indicate the slopes predicted by pseudo-isothermal spheres with core radii of $0.5,1$ and $2 \mathrm{kpc}$, whereas the solid lines represent the Navarro et al. (1996) CDM predictions for characteristic halo radii of 10,20 and $100 \mathrm{kpc}$.

de Blok et al. (2003). This result lends further support for pseudo-isothermal spheres as superior models for the dark halos of these objects.

\section{References}

Bell, E. F., \& de Jong, R. S. 2001, ApJ, 550, 212

de Blok, W. J. G., \& Bosma, A. 2002, A\&A, 385, 816

de Blok, W. J. G., Bosma, A., \& McGaugh, S. 2003, MNRAS, 340, 657

Fuchs, B. 2003, Ap\&SS, 284, 719

McGaugh, S., \& de Blok, W. J. G. 1998, ApJ, 499, 41

Navarro, J. F., Frenk, C. S., \& White, S. D. M. 1996, ApJ, 462, 563

Swaters, R. A., Madore, B. F., van den Bosch, F. C., \& Balcells, M. 2003, ApJ, 583,732 